\title{
Cyclopropanation of alkenes using hypervalent iodine reagents
}

\author{
Andreas S. Biland, ${ }^{a}$ Sabine Altermann, ${ }^{b}$ and Thomas Wirth ${ }^{a, b}$ \\ ${ }^{a}$ Universität Basel, Department Chemie, St. Johanns-Ring 19, 4056 Basel, Switzerland \\ ${ }^{b}$ Cardiff University, Department of Chemistry, PO Box 912, Cardiff CF10 3TB, United Kingdom \\ E-mail:wirth@cf.ac.uk
}

\section{Dedicated to Professor Anastasios Varvoglis on the occasion of his $65^{\text {th }}$ birthday}

(received 10 Jan 03; accepted 01 Apr 03; published on the web 06 June 03)

\begin{abstract}
The addition of bisnucleophiles to alkenes is mediated by hypervalent iodine compounds. This reaction, either performed stepwise or in a one - pot procedure, is a cyclopropanation of alkenes yielding 1,1-dicyanocyclopropane derivatives as reaction products.
\end{abstract}

Keywords: Addition reactions, alkenes, cyclopropanes, electrophiles, hypervalent iodine compounds

\section{Introduction}

Hypervalent iodine compounds are well known for their mild oxidative properties. But a wide range of different reactions using these compounds is possible. ${ }^{1}$ They can be used as electrophilic reagents and various functionalizations of unsaturated systems have been carried out. Oxidative additions to double bonds have been carried out using a wide range of different nucleophiles. Recently the co-introduction of non-equivalent nucleophiles has been investigated intensively. ${ }^{2}$

\section{Results and Discussion}

General Procedures. [Hydroxy(tosyloxy)iodo]benzene was first reported in $1970^{3}$ and is often called Koser's reagent. ${ }^{4}$ This reagent is known to ditosylate alkenes efficiently and one can use either two equivalents of the reagent or one equivalent together with one equivalent paratoluenesulfonic acid. Compound $\mathbf{2}$ is obtained from styrene $\mathbf{1}$ in good yield together with a rearranged side product $\mathbf{3}$. We have replaced either one or both of the tosylates in $\mathbf{3}$ with an azide depending on the reaction conditions. Using chiral [hydroxy(tosyloxy)iodo]arene derivatives we 
were able to synthesize ditosylate 2 in up to $65 \% e e .^{5}$ Being a clean $\mathrm{S}_{\mathrm{N}} 2$ process, the resulting azides $\mathbf{4}$ and $\mathbf{5}$ have the same enantiomeric excess and can be reduced to the corresponding amine like $\mathbf{6}$ easily. ${ }^{6}$

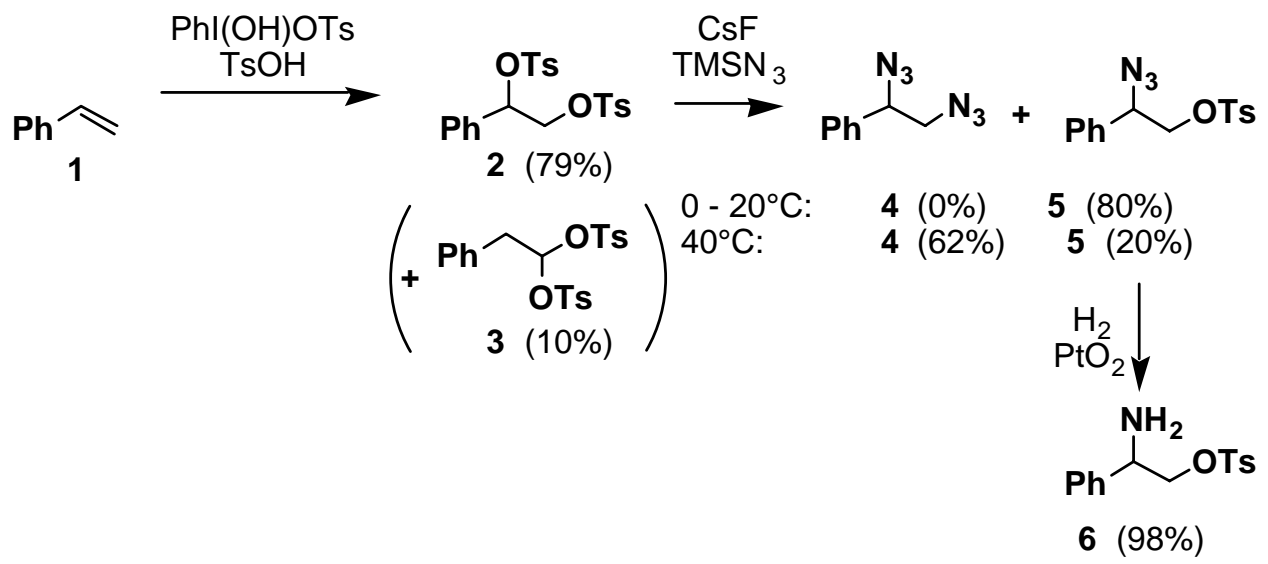

We then explored the possibility of adding bisnucleophiles to compound 2. Addition of acetylacetone 7 lead to the formation of a five-membered heterocycle which has been synthesized earlier by different routes. ${ }^{7}$ After initial attack of the central carbon atom in 7 at the benzylic position, the enolate oxygen of the 1,3-dicarbonyl compound attacks as a second nucleophile to give the dihydrofurane derivative $\mathbf{8}$ in $37 \%$ yield. Malonodinitrile $\mathbf{9}$ as bisnucleophile, however, generated the cyclopropane derivative $\mathbf{1 0}$ in low yields.
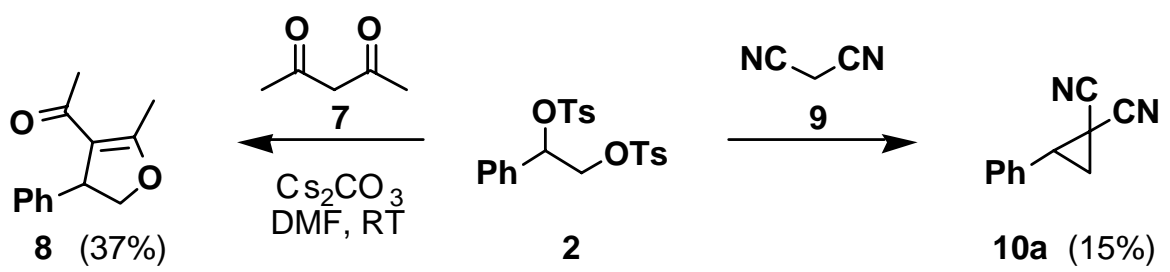

As the electrophilic activation of double bond with (diacetoxyiodo)benzene is known for a long time, we tried to directly introduce the bisnucleophile thus performing the cyclopropanation in one step starting from the corresponding alkene. ${ }^{8}$ We were pleased to find an even higher yields in the corresponding 1,1-dicyanocyclopropane derivatives of type 10. The results are summarized in the following table. Other styrene compounds have been investigated as well, but $\alpha$-methylstyrene and $\beta$-methylstyrene did not yield any product under the reaction conditions.

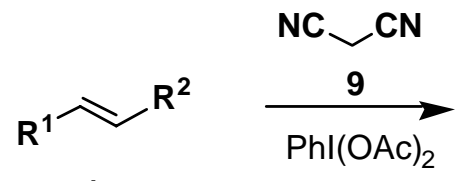

1

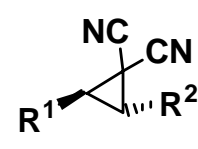

10 
1,1-Dicyanocyclopropanes of type $\mathbf{1 0}$ can be prepared also by other, more complicated routes $^{9}$ and have been used for various photochemical investigations. ${ }^{10}$

Intermolecular $^{11}$ (photochemical) and intramolecular ${ }^{12}$ (thermal) cyclopropanations using iodonium ylides as carbene precursors are known and several examples have been reported. We could not observe any reaction between (diacetoxyiodo)benzene and malonodinitrile under the reaction conditions. Even prolonged heating gave an unchanged ${ }^{1} \mathrm{H}$ NMR spectrum

Table 1. Cyclopropanation of Alkenes

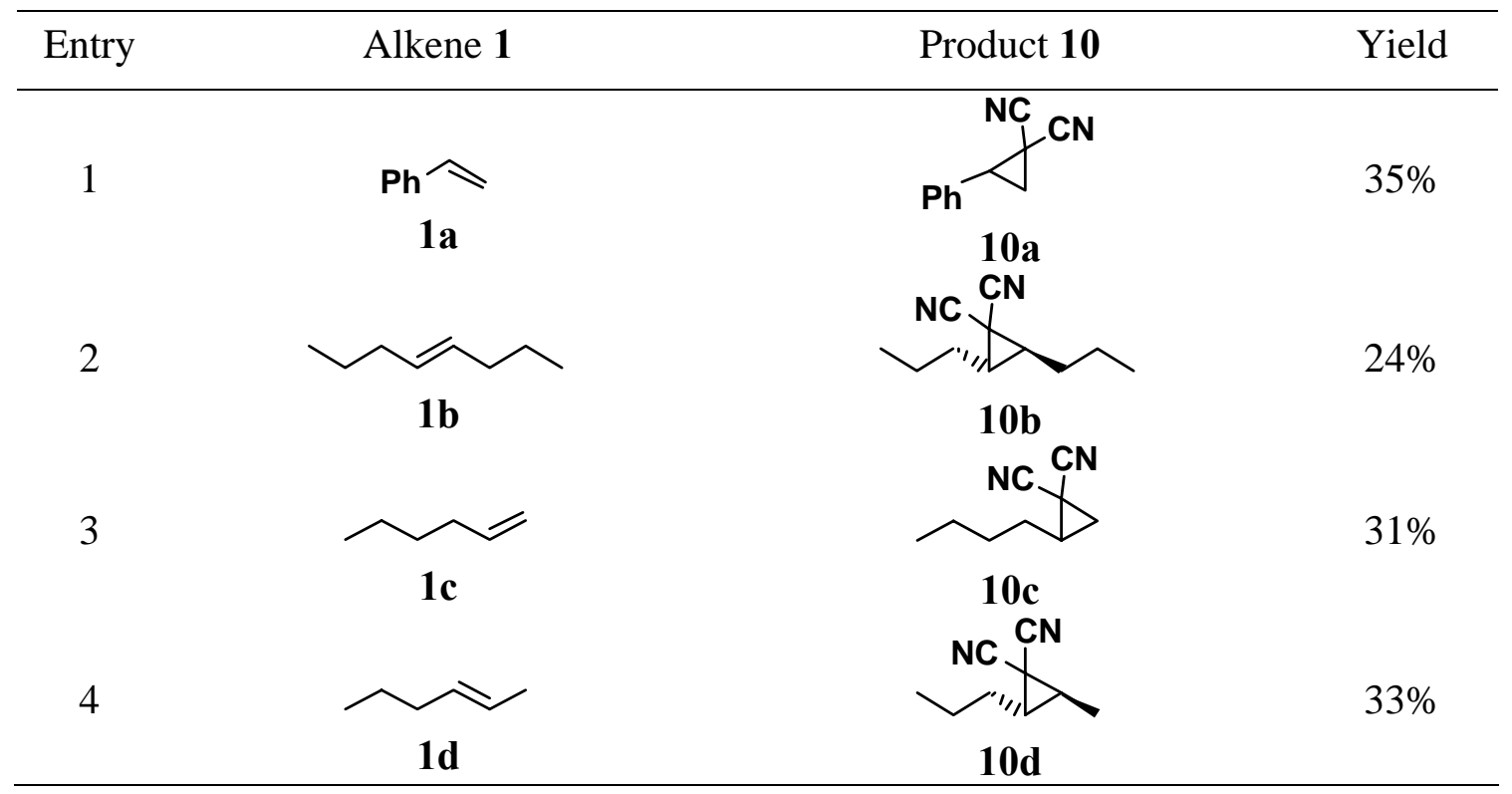

with no indication of iodonium ylide formation. We therefore assume a stepwise reaction as shown below. The malonodinitrile attacking the activated alkene in a first step to yield an intermediate of type 11. The hypervalent iodine species is then acting as a hypernucleofuge allowing the second attack to form the cyclopropane derivative. Within the limits of alkene purities, the cyclopropanation reaction is stereoselective and by NMR analysis no cis-isomers (Table 1 , entries 2 and 4 ) have been detected.

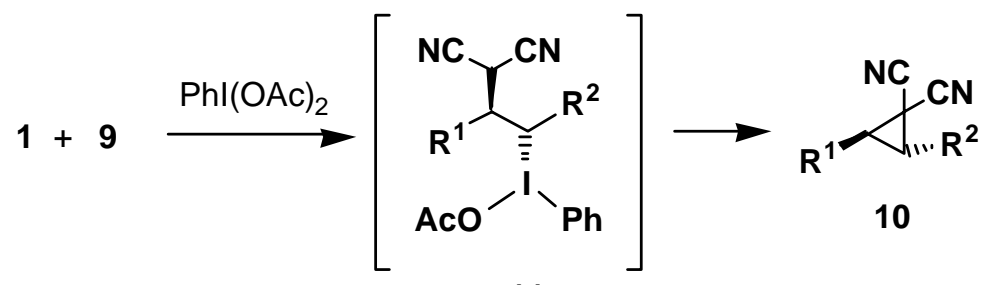

11

In conclusion, we have developed a cyclopropanation of alkenes leading to 1,1,dicyanocyclopropane derivatives. Although the yields are still quite low, work concerning the optimization of the reaction is in progress. 


\section{Experimental Section}

Hydroxy(tosyloxyiodo)benzene (100 mg, $0.26 \mathrm{mmol}$ ) and p-toluenesulfonic acid (46 mg, 0.26 mmol) were dissolved in methylene chloride $(6 \mathrm{~mL})$ and cooled to $0^{\circ} \mathrm{C}$. Styrene $(54 \mathrm{mg}, 0.52$ mmol) is dropwise added to the suspension and after stirring for $28 \mathrm{~h}$ at $\mathrm{rt}$ the solvent was removed and the residue purified by flash-chromatography (tert-butyl methyl ether: pentane 1 : 2). Compound 2 (46 mg, $79 \%$ ) and 3 (6 mg, $10 \%$ ) were obtained.

2. mp.: $117-120{ }^{\circ} \mathrm{C} ;{ }^{1} \mathrm{H}-\mathrm{NMR}\left(300 \mathrm{MHz}, \mathrm{CDCl}_{3}\right): \delta=7.64$ (m, 4H), 7.22 (m, 9H), 5.56 (dd, $J=$ 7.5, $J=4.2, \mathrm{CH}), 4.16\left(\mathrm{~m}, 2 \mathrm{H}, \mathrm{CH}_{2}\right), 2.44$ (s, 3H, $\left.\mathrm{CH}_{3}\right), 2.39$ (s, 3H, $\left.\mathrm{CH}_{3}\right) ;{ }^{13} \mathrm{C}-\mathrm{NMR}(75 \mathrm{MHz}$, $\mathrm{CDCl}_{3}$ ): $\delta=145.1$ (s), 144.8 (s), 133.6 (s), 133.4 (s), 133.3 (s), 129.9 (d, 2C), 129.6 (d, 2C), 129.3 (d), 128.7 (d, 2C), 127.9 (d, 2C), 127.9 (d, 2C), 126.8 (d, 2C), 80.0 (t), 70.1 (d), 21.7 (q), 21.6 (q), IR $\left(\mathrm{CHCl}_{3}\right): v=3373,3037,1600,1497,1370,1263,1176,1136,1051,1016,916$, 867, 814, $562 \mathrm{~cm}^{-1}$; MS(FAB + KCl): 485 (32\%, M + K), 447 (4, M+H), 275 (97), 155 (100), 104 (19), 91 (56), 83 (9), 69 (12), 57 (24), 43(22).

3. mp.: 93 - $96{ }^{\circ} \mathrm{C} ;{ }^{1} \mathrm{H}-\mathrm{NMR}\left(300 \mathrm{MHz}, \mathrm{CDCl}_{3}\right): \delta=7.57$ (m, $\left.4 \mathrm{H}\right), 7.19$ (m, $\left.9 \mathrm{H}\right), 6.37$ (t, $J=5.7$, $1 \mathrm{H}, \mathrm{CH}), 3.11$ (d, $\left.J=5.7,2 \mathrm{H}, \mathrm{CH}_{2}\right), 2.40\left(\mathrm{~s}, 6 \mathrm{H}, \mathrm{CH}_{3}\right) ;{ }^{13} \mathrm{C}-\mathrm{NMR}\left(75 \mathrm{MHz}, \mathrm{CDCl}_{3}\right): \delta=145.1$ (s, 2C), 132.9 (s), 132.3 (s, 2C), 129.7 (d), 129.6 (d, 4C), 128.5 (d, 2C), 127.8 (d, 4C), 127.2 (d), 99.5 (d), 41.9 (t), 21.6 (q, 2C); IR $\left(\mathrm{CHCl}_{3}\right): v$ = 3036, 2929, 1598, 1496, 1455, 1380, 1096, 1072, 1019, 956, 908, 853, $814 \mathrm{~cm}^{-1}$.

Cesium fluoride (81 mg, $0.5 \mathrm{mmol}$ ) under Ar is treated with a solution of trimethylsilyl azide (81 $\mathrm{mg}, 0.7 \mathrm{mmol})$ in DMF (2 mL). After $20 \mathrm{~min}$ stirring at rt a solution of $2(80 \mathrm{mg}, 0.18 \mathrm{mmol})$ in DMF $(2 \mathrm{~mL})$ is added at $0{ }^{\circ} \mathrm{C}$. After stirring for $50 \mathrm{~h}$ at $40{ }^{\circ} \mathrm{C}$ sat. $\mathrm{NaHCO}_{3}(4 \mathrm{~mL})$ and water $(10$ $\mathrm{mL}$ ) are added. After extraction (ethyl acetate, 2 x $20 \mathrm{~mL}$ ) the combined organic phases are dried with magnesium sulfate, the solvent was removed and the residue purified by preparative TLC (tert-butyl methyl ether : pentane $1: 10)$ to obtain compound 4 (21 mg, $62 \%)$ as a yellow oil together with 5 (20\%).

4. ${ }^{1} \mathrm{H}-\mathrm{NMR}$ (300 MHz, $\mathrm{CDCl}_{3}$ ): $\delta=7.35$ (m, 5H), 4.67 (dd, $\left.J=8.2, J=5.2,1 \mathrm{H}, \mathrm{CH}\right) 3.47(\mathrm{~m}$, 2H, $\mathrm{CH}_{2}$ ); ${ }^{13} \mathrm{C}-\mathrm{NMR}\left(75 \mathrm{MHz}, \mathrm{CDCl}_{3}\right.$ ): $\delta=136.3$ (s), 129.1 (d, 2C), 129.0 (d), 126.9 (d, 2C), 65.5 (t), 56.0 (d); IR $\left(\mathrm{CHCl}_{3}\right): v=2927,2492,2104,1439,1454,1018,907 \mathrm{~cm}^{-1}$; MS(EI): 132 (40, M-2N $\mathrm{N}_{2}$ ), 104 (76), 77(100), 63 (7), 51 (35), 39 (9); HPLC: $\lambda=220 \mathrm{~nm}$, hexane : 2-propanol $95: 5,0.5 \mathrm{ml} / \mathrm{min}$, Chiracel OD, $\mathrm{t}_{\mathrm{R} 1}=10.28 \mathrm{~min}, \mathrm{t}_{\mathrm{R} 2}=20.90 \mathrm{~min}$.

Cesium fluoride (160 mg, $1.0 \mathrm{mmol}$ ) under Ar is treated with a solution of trimethylsilyl azide (166 mg, $1.4 \mathrm{mmol}$ ) in DMF (2 mL). After $20 \mathrm{~min}$. stirring at rt a solution of 2 (163 mg, 0.3 mmol) in DMF (2 mL) is added at $0{ }^{\circ} \mathrm{C}$. After stirring for $42 \mathrm{~h}$ at rt sat. $\mathrm{NaHCO}_{3}(4 \mathrm{~mL})$ and water $(10 \mathrm{~mL})$ are added. After extraction (ethyl acetate, $2 \times 20 \mathrm{~mL}$ ) the combined organic phases are dried with magnesium sulfate, the solvent was removed and the residue purified by flash-chromatography (tert-butyl methyl ether: pentane 1: 2) to obtain compound 5 (91 mg, $80 \%)$ as a yellow oil. 
5. ${ }^{1} \mathrm{H}-\mathrm{NMR}\left(300 \mathrm{MHz}, \mathrm{CDCl}_{3}\right): \delta=7.77$ (d, $\left.J=1.8,2 \mathrm{H}\right), 7.29(\mathrm{~m}, 7 \mathrm{H}), 4.77$ (dd, $J=8.5, J=$ 4.4, 1H, CH), 4.21 (m, 2H, $\mathrm{CH}_{2}$ ), 2.44 (s, 3H, $\left.\mathrm{CH}_{3}\right) ;{ }^{13} \mathrm{C}-\mathrm{NMR}\left(75 \mathrm{MHz}, \mathrm{CDCl}_{3}\right.$ ): $\delta=145.1$ (s), 134.6 (s), 132.5 (s), 129.9 (d, 2C), 129.1 (d), 129.0 (d, 2C), 127.9 (d, 2C), 127.0 (d, 2C), 71.6 (t), 63.9 (d), 21.6 (q); IR $\left(\mathrm{CHCl}_{3}\right): v=2109,1599,1454,1367,1177,1097,982 \mathrm{~cm}^{-1}$; MS(FA(B): 318 (13\%, M+H), 290 (19, M-N $), 275$ (27, M- N ${ }_{3}$ ), 155 (37), 137 (85), 118 (68), 104 (30), 91 (100), 77(5), 69 (64), 57 (86), 43 (83), HPLC: $\lambda=254$ nm, hexane : 2-propanol 95 : 5, $0.5 \mathrm{ml} / \mathrm{min}$, Chiracel OD, $\mathrm{t}_{\mathrm{R} 1}=30.48 \mathrm{~min}, \mathrm{t}_{\mathrm{R} 2}=38.72 \mathrm{~min}$.

Compound 5 (65 mg, $0.21 \mathrm{mmol}$ ) and platinum dioxide (1.5 mg) are dissolved in methanol $(1 \mathrm{~mL})$ and trifluoroacetic acid $(2 \mu \mathrm{L})$ and hydrogenated $(1 \mathrm{~atm})$ for $2 \mathrm{~h}$ at $0{ }^{\circ} \mathrm{C}$ and for $18 \mathrm{~h}$ at $\mathrm{rt}$. After filtration (celite) the solvent was removed and $\mathbf{6}$ was obtained (60 mg, 98\%) as a white solid.

6. mp. 102 - $104{ }^{\circ} \mathrm{C} ;{ }^{1} \mathrm{H}-\mathrm{NMR}$ (300 MHz, $\left.\mathrm{CDCl}_{3}, \mathrm{CD}_{3} \mathrm{OD}\right): \delta=7.66$ (m, 2H), 7.25 (m, 7H), 4.55 (m, 1H, CH), 4.35 (m, 2H, CH ), 2.38 (s, 3H, CH $)$; MS(FA(B): 292 (100, M+H), 275 (11), 249 (7), 211 (13), 155 (27), 120 (20), 106 (15), 91 (21), 79 (6), 69 (7), 57 (14), 43 (13).

To a suspension of cesium carbonate $(755 \mathrm{mg}, 2.7 \mathrm{mmol}), 2$ (400 $\mathrm{mg}, 0.9 \mathrm{mmol}$ ) in DMF $(10 \mathrm{~mL})$ was added acetylacetone (453 mg, $4.5 \mathrm{mmol})$. After $15 \mathrm{~h}$ stirring at rt sat. $\mathrm{NaHCO}_{3}$ (5 $\mathrm{mL})$ and water $(30 \mathrm{~mL}$ ) were added. After extraction (ethyl acetate, 3 x $30 \mathrm{~mL}$ ) the combined organic phases are dried with magnesium sulfate, the solvent was removed and the residue purified by flash-chromatography (tert-butyl methyl ether: pentane: 5) to obtain compound 8 (68 $\mathrm{mg}, 37 \%)$ as a colorless oil.

8. ${ }^{1} \mathrm{H}-\mathrm{NMR}\left(300 \mathrm{MHz}, \mathrm{CDCl}_{3}\right.$ ): $\delta=7.26$ (m, 5H), 4.74 (dd, $\left.J=9.0, J=10.1,1 \mathrm{H}, \mathrm{CH}\right), 4.37$ (ddd, $J=5.1 \mathrm{~Hz}, J=10.1 \mathrm{~Hz}, J=0.9 \mathrm{~Hz}, 1 \mathrm{H}, \mathrm{CH}_{2}$ ), 4.27 (dd, $J=5.1 \mathrm{~Hz}, J=9.0 \mathrm{~Hz}, 1 \mathrm{H}, \mathrm{CH}_{2}$ ), $2.36 \mathrm{ppm}\left(\mathrm{d}, J=0.9 \mathrm{~Hz}, 3 \mathrm{H}, \mathrm{CH}_{3}-\mathrm{C}=\mathrm{C}\right), 1.58 \mathrm{ppm}\left(\mathrm{s}, 3 \mathrm{H}, \mathrm{CH}_{3}\right) ;{ }^{13} \mathrm{C}-\mathrm{NMR}\left(75 \mathrm{MHz}, \mathrm{CDCl}_{3}\right): \delta$ = 194.9 (s), 169.6 (s), 143.6 (s), 128.8 (d, 2C), 127.1 (d, 2C), 127.1 (d) , 116.1 (s), 78.8 (t), 48.9 (d), 29.6 (q), 15.0 (q) / IR $\left(\mathrm{CHCl}_{3}\right)$ : 3006, 1662, 1587, 1492, 1454, 1383, 1322, 1126, 1079, 998, 939, 917, $628 \mathrm{~cm}^{-1}$; MS 202 (100, M), 159 (30), 145 (39), 128 (20), 115 (33), 91 (20), 77 (21), 65 (11), 63 (12), 51 (25); HPLC: $\lambda=278 \mathrm{~nm}$, hexane: 2-propanol 95 : 5, $0.5 \mathrm{ml} / \mathrm{min}$, Chiracel $\mathrm{OD}, \mathrm{t}_{\mathrm{R} 1}=19.30 \mathrm{~min}, \mathrm{t}_{\mathrm{R} 2}=23.55 \mathrm{~min}$.

(Diacetoxyiodo)benzene (300 mg,0.93 mmol) and malono dinitrile (92 $\mathrm{mg}, 1.4 \mathrm{mmol}$ ) are dissolved in methylene chloride $(4 \mathrm{~mL})$ and the alkene $(1.86 \mathrm{mmol})$ is added at $-78{ }^{\circ} \mathrm{C}$. After stirring for $24 \mathrm{~h}$ at $\mathrm{rt}$ the solvent is removed the residue purified by flash-chromatography (tertbutyl methyl ether : pentane $1: 5$ ) to obtain compound $\mathbf{1 0 .}$

Alkene: styrene, 10a: ${ }^{1} \mathrm{H}-\mathrm{NMR}\left(300 \mathrm{MHz}, \mathrm{CDCl}_{3}\right): \delta=7.43(\mathrm{~m}, 3 \mathrm{H}), 7.29(\mathrm{~m}, 2 \mathrm{H}), 3.30(\mathrm{t}, J=$ 9.1, 1H), 2.26 (dd, $J=9.1, J=1.0,2 \mathrm{H}, \mathrm{CH}_{2}$ ); ${ }^{13} \mathrm{C}-\mathrm{NMR}$ (75 MHz, $\mathrm{CDCl}_{3} / \mathrm{TMS}$ ), $\delta=130.5$ (s), 129.5 (d), 129.2 (d, 2C), 128.3 (d, 2C), 115.3 (s), 113.0 (s), 35.1 (d), 29.7 (t), 22.4 (s); IR $\left(\mathrm{CHCl}_{3}\right): v=3029,2400,2250,1521,1474,1421,1045,1029,927 \mathrm{~cm}^{-1}$; MS(EI): 168 (100\%, M), 140 (57), 114 (28), 103 (9), 89 (11), 77 (20), 63 (13), 51 (21); HPLC: $\lambda=220$ nm; hexane : 2-propanol $80: 20,0.5 \mathrm{ml} / \mathrm{min}$, Chiracel OD, $\mathrm{t}_{\mathrm{R} 1}=47.47 \mathrm{~min}, \mathrm{t}_{\mathrm{R} 2}=62.03 \mathrm{~min}$. 
Alkene: E-4-octene, 10b: ${ }^{1} \mathrm{H}-\mathrm{NMR}$ (400 MHz, $\left.\mathrm{CDCl}_{3}\right): \delta=1.55(\mathrm{~m}, 10 \mathrm{H}), 0.95(\mathrm{t}, J=7,6 \mathrm{H})$;

${ }^{13} \mathrm{C}-\mathrm{NMR}\left(100 \mathrm{MHz}, \mathrm{CDCl}_{3} / \mathrm{TMS}\right), \delta=116.7$ (s, 2C), 38.0 (d, 2C), 35.7 (s), 32.3 (t, 2C), 21.7 (t, 2C), 14.0 (q, 2C); IR $\left(\mathrm{CHCl}_{3}\right): v=3021,2966,2885,2243,1464,1383,1222 \mathrm{~cm}^{-1}$.

Alkene: 1-hexene, 10c: ${ }^{1} \mathrm{H}-\mathrm{NMR}\left(400 \mathrm{MHz}, \mathrm{CDCl}_{3}\right): \delta=0.89\left(\mathrm{t}, J=5.1,3 \mathrm{H}, \mathrm{CH}_{3}\right), 1.3-1.6(\mathrm{~m}$, 7H), 1.89 (m, 2H); ${ }^{13} \mathrm{C}-\mathrm{NMR}\left(100 \mathrm{MHz}, \mathrm{CDCl}_{3} / \mathrm{TMS}\right), \delta=112.7$ (s, 2C), 37.0, 33.7, 31.1, 30.4, 19.9, 13.4, 10.9; IR $\left(\mathrm{CHCl}_{3}\right): v=2945,2855,2243,1453 \mathrm{~cm}^{-1}$.

Alkene: E-2-hexene, 10d: ${ }^{1} \mathrm{H}-\mathrm{NMR}\left(400 \mathrm{MHz} \mathrm{CDCl}_{3}\right): \delta=0.85$ (t, $\left.J=4.8,3 \mathrm{H}, \mathrm{CH}_{3}\right), 1.35$ (d, $J$ $\left.=6.16,3 \mathrm{H}, \mathrm{CHCH}_{3}\right), 1.50(\mathrm{~m}, 5 \mathrm{H}), 1.71(\mathrm{~m}, 1 \mathrm{H}) ;{ }^{13} \mathrm{C}-\mathrm{NMR}\left(100 \mathrm{MHz}, \mathrm{CDCl}_{3} / \mathrm{TMS}\right), \delta=$ 114.6 (s, 2C), 38.9, 32.9, 32.2, 31.3, 21.7, 15.2, 13.9; IR $\left(\mathrm{CHCl}_{3}\right): v=2966,2865,2243,1461$, 1383, $1257,1057 \mathrm{~cm}^{-1}$.

\section{Acknowledgements}

Funding was provided by the Schweizer Nationalfonds and by Cardiff University.

\section{References}

1. Hypervalent Iodine Chemistry, Wirth, T. Ed. Top. Curr. Chem. 2003, 224.

2. Koser, G. F. Top. Curr. Chem. 2003, 224, 137.

3. Neilands, O.; Karele, B. Zh. Org. Khim. 1970, 6, 885.

4. (a) Koser, G. F.; Wettach, R. H.; Troup, J. M.; Frenz, B. A. J. Org. Chem. 1976, 41, 3609.

(b) Koser, G. F.; Wettach, R. H. J. Org. Chem. 1977, 42, 1476.

5. $\quad$ Hirt, U. H.; Spingler, B.; Wirth, T. J. Org. Chem. 1998, 63, 7674.

6. Compound $\mathbf{6}$ was synthesized also by a different route from phenylglycinol: Sakai, N.; Ohfune, Y. J. Am. Chem. Soc. 1992, 114, 998.

7. (a) Ichikawa, K.; Uemura, S.; Sugita, T. Tetrahedron 1966, 22, 407. (b) Johnson, C. R.; Lockard, J. P.; Kennedy, E. R. J. Org. Chem. 1980, 45, 264.

8. Hatzigrigoriou, E.; Bakola-Christianopoulou, M.; Varvoglis, A. J. Chem. Res. (S) 1987, 374.

9. Stevens, C. V.; Van Heecke, G.; Barbero, C.; Patora, K.; De Kimpe, N.; Verhé, R. Synlett 2002, 1089.

10. (a) Leitich, J.; Ritter-Thomas, U.; Heise, I. J. Photochem. Photobiol. A: Chemistry 2002, 147, 177. (b) Leitich, J.; Ritter-Thomas, U.; Heise, I.; Tsay, Y.; Rust, J. J. Photochem. Photobiol. A: Chemistry 2002, 147, 157.

11. (a) Hadjiarapoglou, L.; Spyroudis, S.; Varvoglis, A. J. Am. Chem. Soc. 1985, 107, 7178. (b) Hadjiarapoglou, L.; Varvoglis, A.; Alcock, N. W.; Pike, G. A. J. Chem. Soc., Perkin Trans. 1 1988, 2839. (c) Camacho, M. B.; Clark, A. E.; Liebrecht, T. A.; DeLuca, J. P. J. Am. Chem. Soc. 2000, 122, 5210.

12. (a) Moriarty, R. M.; Kim, J.; Guo, L. Tetrahedron Lett. 1993, 34, 4129. (b) Moriarty, R. M.; May, E. J.; Guo, L.; Prakash, O. Tetrahedron Lett. 1998, 39, 765. 